\title{
REFERENCES
}

1. G. Ascoli, Revista Univ. Nac. de Tucumán, Serie A : Mat. y Fis. Teór, 2 (1941), 131-140.

2. G. Ascoli, Annali di Mat. Pur. Appl., (4) 26 (1947), 199-206.

3. N. Levinson, Duke Math. J., 15 (1948), 111-126.

4. F. V. Atkinson, Annali di Mat. Pur. Appl., (4) 37 (1954), 347-378.

5. A. Wintner, Phys. Rev., 72 (1947), 516-517. $71-87$.

6. F. V. Atkinson, Univ. Nac. del Tucumán, Revista, Serie A, Mat. y Fis. Teór., 8 (1951),

7. P. Hartman and A. Wintner, Amer. J. Math., 77 (1955), 45-86.

8. R. Bellman, Stability theory of differential equations (New York, 1953).

9. R. Bellman, Duke Math. J., 22 (1955), 511-513.

10. L. A. Gusarov, Moskov. Gos. Univ. Uč. Zap., 165, Mat. 7 (1954), 223-237.

11. I. M. Sobol', Mat. Sbomik (N.S.) 28 (70) (1951), 707-714.

The Canberra University College, Canberra

Australian Capital Territory

Austratia

\section{INFINITE INTEGRALS INVOLVING PRODUCTS OF LEGENDRE FUNCTIONS}

\author{
by K. C. SHARMA
}

(Received 1st November, 1956)

1. In this paper we evaluate a few infinite integrals involving products of Legendre functions. The results obtained herein are quite general and include, as particular cases, some known results.

We shall evaluate these integrals with the help of a theorem in operational calculus proved in $\S 2$.

We write

when

$$
\psi(p) \fallingdotseq f(x)
$$

$$
\psi(p)=p \int_{0}^{\infty} e^{-p x} f(x) d x
$$

and

$$
\phi(p) \frac{\underline{K}}{\bar{K}} f(x)
$$

when

$$
\phi(p)=(2 / \pi)^{\mathfrak{l}} p \int_{0}^{\infty}(p x)^{\natural} K_{\nu}(p x) f(x) d x .
$$

Formula (2) is a generalisation of (1) as given by Meijer [7] and it reduces to (1) when $\nu= \pm \frac{1}{2}$, since

$$
K_{ \pm \mathbf{1}}(x)=\left(\frac{\pi}{2 x}\right)^{\frac{1}{2}} e^{-x}
$$


2. TheOREM. If

$$
\psi(p) \fallingdotseq f(x)
$$

and

$$
\phi(p) \frac{K}{\bar{K}} x^{\mu-1} f(x)
$$

then

$$
\phi(p)=p^{1-\mu} \int_{1}^{\infty}\left(x^{2}-1\right)^{-\frac{1}{1} \mu} \psi(p x) x^{-1} P_{\nu-1}^{\mu}(x) d x,
$$

provided that the integral is convergent and $R(\mu)<1$.

Proof. We know that, if

$$
\psi(p) \fallingdotseq f(x)
$$

then $[1$, p. 129]

$$
p \frac{\psi(p+a)}{p+a} \fallingdotseq e^{-a x} f(x) .
$$

Also $[1, p .278]$

$$
p^{\mu+\frac{1}{2}} e^{a p} K_{\nu}(a p) \fallingdotseq\left(\frac{\pi}{2 a}\right)^{\frac{1}{2}}\left(t^{2}+2 a t\right)^{-\frac{1}{2} \mu} P_{\nu-1}^{\mu}\left(1+\frac{t}{a}\right) .
$$

Using the relations (4) and (5) in Goldstein's result [4] that, if

then

$$
h_{1}(p) \fallingdotseq g_{1}(x) \text { and } h_{2}(p) \fallingdotseq g_{2}(x) \text {, }
$$

$$
\int_{0}^{\infty} h_{1}(x) g_{2}(x) x^{-1} d x=\int_{0}^{\infty} h_{2}(x) g_{1}(x) x^{-1} d x
$$

and then replacing $a$ by $p$, we get the relation

$$
\phi(p)=p \int_{0}^{\infty}\left(t^{2}+2 p t\right)^{-\frac{1}{2} \mu}(p+t)^{-1} \psi(p+t) P_{\nu-\frac{1}{2}}^{\mu}(1+t / p) d t .
$$

Equation (3) follows from (6) when we substitute $t=p(x-1)$.

A particular case of this theorem, when $\mu=\frac{1}{2}$, has been given by Rathie [9] in a slightly different form.

3. We now proceed to evaluate a few infinite integrals by applying the above theorem. In what follows we have used MacRobert's definition of $Q_{n}^{m}(x)$.

(i) From $[9$, p. 176] we have

$$
\begin{aligned}
f(x) & =x^{m-\frac{1}{1}} I_{n+\frac{1}{1}}(x) \\
& \fallingdotseq(2 / \pi)^{\frac{1}{2}} p\left(p^{2}-1\right)^{-\frac{1}{2} m} Q_{n}^{m}(p) \quad \ldots \ldots \ldots \ldots \ldots \\
& \fallingdotseq \psi(p) \quad[R(m+n+1)>0, R(p)>1]
\end{aligned}
$$

and

$$
\begin{aligned}
x^{\mu-1} f(x) & =x^{\mu+m-\frac{3}{2}} I_{n+\frac{1}{3}}(x) \\
& \frac{K}{\bar{K}} p^{1-n-m-\mu} 2^{\mu+m-\frac{3}{2}} \frac{\Gamma\left(\frac{1}{2} \mu+\frac{1}{2} m+\frac{1}{2} \nu+\frac{1}{2} n+\frac{1}{4}\right) \Gamma\left(\frac{1}{2} \mu+\frac{1}{2} m+\frac{1}{2} n-\frac{1}{2} \nu+\frac{1}{4}\right)}{\Gamma\left(\frac{1}{2}\right) \Gamma\left(n+\frac{3}{2}\right)} \\
& \quad{ }_{2} F_{1}\left[\frac{1}{2} \mu+\frac{1}{2} m+\frac{1}{2} n+\frac{1}{2} \nu+\frac{1}{4}, \frac{1}{2} \mu+\frac{1}{2} m+\frac{1}{2} n-\frac{1}{2} \nu+\frac{1}{4} ; n+\frac{3}{2} ; p^{-2}\right] \\
= & \phi(p) \quad\left[R\left(\mu+m+n \pm \nu+\frac{1}{2}\right)>0\right] .
\end{aligned}
$$

using the relations (7) and (8) in (3) and then replacing $\nu$ by $\nu+\frac{1}{2}$, we get 


$$
\begin{aligned}
& \int_{1}^{\infty}\left(x^{2}-1\right)^{-1 \mu}\left(p^{2} x^{2}-1\right)^{-\frac{1}{2} m} Q_{n}^{m}(p x) P_{\nu}^{\mu}(x) d x \\
& =2^{\mu+m-2} p^{-n-m-1} \frac{\Gamma\left({ }_{2}^{1} \mu+\frac{1}{2} m+\frac{1}{2} \nu+\frac{1}{2} n+\frac{1}{2}\right) \Gamma\left(\frac{1}{2} \mu+\frac{1}{2} m-\frac{1}{2} \nu+\frac{1}{2} n\right)}{\Gamma\left(n+\frac{3}{2}\right)} \\
& \quad \times{ }_{2} F_{1}\left[{ }_{2}^{1} \mu+\frac{1}{2} m+\frac{1}{2} \nu+\frac{1}{2} n+\frac{1}{2}, \frac{1}{2} \mu+\frac{1}{2} m-\frac{1}{2} \nu+\frac{1}{2} n ; n+\frac{3}{2} ; p^{-2}\right] .
\end{aligned}
$$

This, by a simple substitution $x=\cosh \theta$, can be expressed in the form

$$
\begin{aligned}
& \int_{0}^{\infty}(\sinh \theta)^{1-\mu}\left(p^{2} \cosh ^{2} \theta-1\right)^{-1} m Q_{n}^{m}(p \cosh \theta) P_{\nu}^{\mu}(\cosh \theta) d \theta \\
& =2^{\mu+m-2} p^{-n-m-1} \frac{\Gamma\left(\frac{1}{2} \mu+\frac{1}{2} m+\frac{1}{2} \nu+\frac{1}{2} n+\frac{1}{2}\right) \Gamma\left(\frac{1}{2} \mu+\frac{1}{2} m-\frac{1}{2} \nu+\frac{1}{2} n\right)}{\Gamma\left(n+\frac{3}{2}\right)} \\
& \quad{ }_{2} F_{1}\left[\frac{1}{2} \mu+\frac{1}{2} m+\frac{1}{2} n+\frac{1}{2} \nu+\frac{1}{2}, \frac{1}{2} \mu+\frac{1}{2} m+\frac{1}{2} n-\frac{1}{2} \nu ; n+\frac{3}{2} ; p^{-2}\right] .
\end{aligned}
$$

Results (9) and (10) are valid for $R(\mu)<1, R(\mu+m+n+\nu+1)>0, R(\mu+m-\nu+n)>0$ and $|p|>1$.

A few particular cases of (9) and (10) are worth mentioning and are given below.

(a) When $p \rightarrow 1$, then, by virtue of the relation

$$
{ }_{2} F_{1}(a, b ; c ; 1)=\frac{\Gamma(c) \Gamma(c-a-b)}{\Gamma(c-a) \Gamma(c-b)} \quad[R(c-a-b)>0]
$$

(10) reduces to

$$
\begin{aligned}
& \int_{0}^{\infty}(\sinh \theta)^{1-\mu-m} Q_{n}^{m}(\cosh \theta) P_{\nu}^{\mu}(\cosh \theta) d \theta \\
& \quad=2^{\mu+m-2} \frac{\Gamma\left(\frac{1}{2} \mu+\frac{1}{2} m+\frac{1}{2} n+\frac{1}{2} \nu+\frac{1}{2}\right) \Gamma\left(\frac{1}{2} \mu+\frac{1}{2} m-\frac{1}{2} \nu+\frac{1}{2} n\right) \Gamma(1-\mu-m)}{\Gamma\left(\frac{1}{2} n-\frac{1}{2} \mu-\frac{1}{2} m-\frac{1}{2} \nu+1\right) \Gamma\left(\frac{1}{2} n-\frac{1}{2} \mu-\frac{1}{2} m+\frac{1}{2} \nu+\frac{3}{2}\right)},
\end{aligned}
$$

where $R(1-\mu-m)>0, R(\mu+m+n-\nu)>0, R(\mu+m+n+\nu+1)>0$ and $R(\mu)<1$.

If we put $\nu=-\mu$ in (10) and (12) and use the result [3, p. 150]

$$
P_{-\mu}^{\mu}(z)=2^{\mu}\left(z^{2}-1\right)^{-1 \mu} / \Gamma(1-\mu)
$$

we get results given by MacRobert [5, p. 95].

Also, when we take $n=m-1$ in (12), then, by virtue of $[6, p .403]$

$$
Q_{m-1}^{m}(z)=2^{m-1} \Gamma(m)\left(z^{2}-1\right)^{-\frac{1}{2} m},
$$

we get another result given by MacRobert [5, p. 96].

(b) Since $[3$, p. 125]

$$
\Gamma(1-\mu) P_{\nu}^{\mu}(z)=2^{-\nu}(z+1)^{1 \mu+\nu}(z-1)^{-\frac{1}{2} \mu}{ }_{2} F_{1}(-\nu,-\nu-\mu ; 1-\mu ;(z-1) /(z+1)),
$$

by taking $\nu=n$ in (9) we get

$$
\begin{aligned}
& \int_{1}^{\infty}\left(x^{2}-1\right)^{-\frac{1}{1} \mu}\left(p^{2} x^{2}-1\right)^{-\frac{1}{2} m} Q_{n}^{m}(p x) P_{n}^{\mu}(x) d x \\
& =2^{\mu+m-2} p^{\mu-\frac{1}{1}}\left(p^{2}-1\right)^{-\frac{1}{2} \mu-\frac{1}{2} m} \Gamma\left(\frac{1}{2} \mu+\frac{1}{2} m\right) \Gamma\left(\frac{1}{2} \mu+\frac{1}{2} m+n+\frac{1}{2}\right) P_{-\frac{1}{2} \mu-\frac{1}{1} m}^{-n-1}\left(\frac{p^{2}+1}{p^{2}-1}\right),
\end{aligned}
$$

where $R(\mu)<1, R(\mu+m+2 n+1)>0, R(\mu+m)>0$ and $|p|>1$.

(c) If we take $\mu=\frac{1}{2}-m$ and use the expansion [3, p. 127]

$$
\Gamma(1-\mu) P_{v}^{\mu}(z)=2^{\mu}\left(z^{2}-1\right)-{ }_{2} F_{1}\left[\frac{1}{2}+\frac{1}{2} \nu-\frac{1}{2} \mu,-\frac{1}{2} \nu-\frac{1}{2} \mu ; 1-\mu ; 1-z^{2}\right],
$$


relation (9) yields the result

$$
\begin{aligned}
& \int_{1}^{\infty}\left(x^{2}-1\right)^{\frac{1}{m} m-\frac{1}{1}}\left(p^{2} x^{2}-1\right)^{-\frac{1}{2} m} Q_{n}^{m}(p x) P_{\nu}^{+-m}(x) d x \\
& \quad=(-1)^{\frac{1}{2} n+\frac{1}{2}} 2^{n-1} p^{-m-\frac{1}{2}} \Gamma\left(\frac{1}{2} n+\frac{1}{2} \nu+\frac{3}{4}\right) \Gamma\left(\frac{1}{2} n-\frac{1}{2} \nu+\frac{1}{4}\right) P_{\nu}^{-n-1}\left(\sqrt{ }\left(1-p^{-2}\right)\right),
\end{aligned}
$$

where $R\left(m+\frac{1}{2}\right)>0, R(m+\nu+1)>0, R\left(n-\nu+\frac{1}{2}\right)>0$ and $|p|>1$.

(d) If we substitute $\frac{1}{2}-\mu-m$ for $\nu$ in (9) and apply [3, p. 129]

$$
\begin{aligned}
\Gamma(1-\mu) P_{v}^{\mu}(z)=2^{\mu}\left(z^{2}-1\right)^{-\frac{1}{2} \mu}\left\{z+\sqrt{ }\left(z^{2}-1\right)\right\}^{\nu+\mu} & \\
& \times{ }_{2} F_{1}\left[-\nu-\mu, \frac{1}{2}-\mu ; 1-2 \mu ; 2 \sqrt{ }\left(z^{2}-1\right) /\left\{z+\sqrt{ }\left(z^{2}-1\right)\right\}\right] .
\end{aligned}
$$

and Whipple's transformation [3, p. 141]

we get

$$
Q_{\nu}^{\mu}(z)=(2 / \pi)^{-\dagger}\left(z^{2}-1\right)^{-1} \Gamma(\nu+\mu+1) P_{-\mu-1}^{-\nu-1}\left\{z / \sqrt{ }\left(z^{2}-1\right)\right\},
$$

$$
\begin{aligned}
& \int_{1}^{\infty}\left(x^{2}-1\right)^{-1 \mu}\left(p^{2} x^{2}-1\right)^{-\frac{1}{m} m} Q_{n}^{m}(p x) P_{t-\mu-m}^{\mu}(x) d x
\end{aligned}
$$

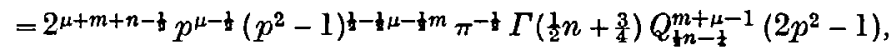

where $R(\mu)<1, R\left(\mu+m+\frac{1}{2} n-\frac{1}{4}\right)>0, R\left(n+\frac{3}{2}\right)>0$ and $|p|>1$.

(e) Since

and [3, p. 129]

$$
\Gamma(1-\mu) P_{0}^{\mu}(z)=(z+1)^{\frac{1}{\mu}}(z-1)^{-\frac{1}{2} \mu}
$$

$$
\Gamma(1-\mu) P_{\nu}^{\mu}(z)=2^{\mu}\left(z^{2}-1\right)^{-\frac{1}{2} \mu} z^{\mu+\nu}{ }_{2} F_{1}\left[-\frac{1}{2} \mu-\frac{1}{2} \nu, \frac{1}{2}-\frac{1}{2} \mu-\frac{1}{2} \nu ; 1-\mu ; 1-z^{-2}\right],
$$

if we put $\nu=0$ in (9), apply the transformation (20) and duplication formula for gamma functions, namely

we get

$$
2^{2 z-1} \Gamma(z) \Gamma\left(z+\frac{1}{2}\right)=\sqrt{\pi} \Gamma(2 z)
$$

$$
\begin{aligned}
\int_{1}^{\infty}(x-1)^{-\mu}\left(p^{2} x^{2}-1\right)^{-\frac{1}{m} m} Q_{n}^{m} & (p x) d x \\
& =p^{\mu-1} \Gamma(1-\mu)\left(p^{2}-1\right)^{1-1 \mu-\frac{1}{1} m} Q_{n}^{\mu+m-1}(p),
\end{aligned}
$$

where $R(\mu)<1, R(\mu+m+n)>0$ and $|p|>1$.

(ii) Now we take $[9$, p. 174]

$$
\begin{aligned}
f(x) & =x^{\alpha-\frac{1}{3}} K_{\gamma+\frac{1}{2}}(x) \\
& \fallingdotseq(\pi / 2)^{1 / 2} \Gamma(\alpha-\gamma) \Gamma(\alpha+\gamma+1) p\left(p^{2}-1\right)^{-1 \alpha} P_{\gamma}^{-\alpha}(p) \\
& =\psi(p),
\end{aligned}
$$

where $R(\alpha+\gamma+1)>0, R(\alpha-\gamma)>0, R(p)>-1$, and

$x^{\mu-1} f(x)=x^{\mu+\alpha-\frac{3}{2}} K_{\gamma+\frac{1}{2}}(x)$

$$
\frac{R}{\bar{K}} \frac{2^{\mu+\alpha-\frac{s}{3}}}{p^{\mu+\alpha+\gamma-1}}
$$

$\times \frac{\Gamma\left[\frac{1}{2}\left(\mu+\alpha+\nu+\gamma+\frac{1}{2}\right)\right] \Gamma\left[\frac{1}{2}\left(\mu+\alpha-\nu+\gamma+\frac{1}{2}\right)\right] \Gamma\left[\frac{1}{2}\left(\mu+\alpha+\nu-\gamma-\frac{1}{2}\right)\right] \Gamma\left[\frac{1}{2}\left(\mu+\alpha-\nu-\gamma-\frac{1}{2}\right)\right]}{\Gamma\left(\frac{1}{2}\right) \Gamma(\mu+\alpha)}$

$\times{ }_{2} F_{1}\left[\frac{1}{2} \mu+\frac{1}{2} \alpha+\frac{1}{2} \nu+\frac{1}{2} \gamma+\frac{1}{4}, \frac{1}{2} \mu+\frac{1}{2} \alpha-\frac{1}{2} \nu+\frac{1}{2} \gamma+\frac{1}{4} ; \mu+\alpha ; 1-p^{-2}\right]$

$=\phi(p)$,

where $R\left(\mu+\alpha \pm \nu+\gamma+\frac{1}{2}\right)>0, R\left(\mu+\alpha \pm \nu-\gamma-\frac{1}{2}\right)>0$, and $\left|1-p^{-2}\right|<1$. 
Applying the theorem and replacing $\nu$ by $\nu+\frac{1}{2}$, we get

$$
\begin{aligned}
& \int_{1}^{\infty}\left(x^{2}-1\right)^{-\frac{1}{1} \mu}\left(p^{2} x^{2}-1\right)^{-\frac{1}{2} \alpha} P_{\gamma}^{-\alpha}(p x) P_{\nu}^{\mu}(x) d x \\
& =\frac{\Gamma[1 / 2(\mu+\alpha+\nu+\gamma+1)] \Gamma\left[\frac{1}{2}(\mu+\alpha-\nu+\gamma)\right] \Gamma\left[\frac{1}{2}(\mu+\alpha+\nu-\gamma)\right] \Gamma\left[\frac{1}{2}(\mu+\alpha-\nu-\gamma-1)\right]}{\pi \Gamma(\mu+\alpha) \Gamma(\alpha-\gamma) \Gamma(\alpha+\gamma+1)} \\
& \times \frac{2^{\mu+\alpha-2}}{p^{\alpha+\gamma+1}} F_{1}\left[\frac{1}{2} \mu+\frac{1}{2} \alpha+\frac{1}{2} \nu+\frac{1}{2} \gamma+\frac{1}{2}, \frac{1}{2} \mu+\frac{1}{2} \alpha-\frac{1}{2} \nu+\frac{1}{2} \gamma ; \mu+\alpha ; 1-p^{-2}\right],
\end{aligned}
$$

where $R(\mu)<1, R(\mu+\alpha+\nu+\gamma+1)>0, R(\mu+\alpha-\nu+\gamma)>0, R(\mu+\alpha+\nu-\gamma)>0$,

$R(\mu+\alpha-\nu-\gamma-1)>0$, and $\left|1-p^{-2}\right|<1$.

If we substitute $x=\cosh \theta,(28)$ takes the form

$$
\begin{gathered}
\int_{0}^{\infty}(\sinh \theta)^{1-\mu}\left(p^{2} \cosh ^{2} \theta-1\right)^{-\frac{1}{2} \alpha} P_{\gamma}^{-\alpha}(p \cosh \theta) P_{\nu}^{\mu}(\cosh \theta) d \theta \\
=\frac{2^{\mu+\alpha-2}}{p^{\alpha+\gamma+1}} \cdot \frac{\Gamma\left[\frac{1}{2}(\mu+\alpha+\nu+\gamma+1)\right] \Gamma\left[\frac{1}{2}(\mu+\alpha-\nu+\gamma)\right] \Gamma\left[\frac{1}{2}(\mu+\alpha+\nu-\gamma)\right] \Gamma\left[\frac{1}{2}(\mu+\alpha-\nu-\gamma-1)\right]}{\pi \Gamma(\mu+\alpha) \Gamma(\alpha-\gamma) \Gamma(\alpha+\gamma+1)} \\
\quad{ }_{2} F_{1}\left[\frac{1}{2} \mu+\frac{1}{2} \alpha+\frac{1}{2} \nu+\frac{1}{2} \gamma+\frac{1}{2}, \frac{1}{2} \mu+\frac{1}{2} \alpha-\frac{1}{2} \nu+\frac{1}{2} \gamma ; \mu+\alpha ; 1-p^{-2}\right], \quad \ldots \ldots \ldots \ldots \ldots . . .(29)
\end{gathered}
$$

where $R(\mu)<1, R(\mu+\alpha+\nu+\gamma+1)>0, R(\mu+\alpha-\nu+\gamma)>0, R(\mu+\alpha+\nu-\gamma)>0$,

$$
R(\mu+\alpha-\nu-\gamma-1)>0 \text { and }\left|1-p^{-2}\right|<1 \text {. }
$$

We mention some particular cases of (28) and (29). A few of them, which express Legendre functions as infinite integrals involving Legendre functions are quite interesting.

(a) When we assume $p=1,(28)$ yields

$$
\begin{gathered}
\int_{1}^{\infty}\left(x^{2}-1\right)^{-\frac{1}{1} \mu-\frac{1}{\alpha} \alpha} P_{\gamma}^{-\alpha}(x) P_{\nu}^{\mu}(x) d x \\
=2^{\mu+\alpha-2} \frac{\Gamma\left[\frac{1}{2}(\mu+\alpha+\nu+\gamma+1)\right] \Gamma\left[\frac{1}{2}(\mu+\alpha-\nu+\gamma)\right] \Gamma\left[\frac{1}{2}(\mu+\alpha+\nu-\gamma)\right] \Gamma\left[\frac{1}{2}(\mu+\alpha-\nu-\gamma-1)\right]}{\pi \Gamma(\mu+\alpha) \Gamma(\alpha-\gamma) \Gamma(\alpha+\gamma+1)},
\end{gathered}
$$

where $\quad R(\mu)<1, R(\mu+\alpha+\nu+\gamma+1)>0, R(\mu+\alpha-\nu+\gamma)>0, R(\mu+\alpha+\nu-\gamma)>0$

and $\quad R(\mu+\alpha-\nu-\gamma-1)>0$.

If we take $\nu=-\mu=\lambda+\frac{1}{2} \alpha-1$ in (30) and apply the formulae (13) and (24) we get a known result $[2$, p. 320].

Similarly if we take $\nu=-\mu$ in (29) and apply the relations (13) and (24) we get a result given by MacRobert [5, p. 96].

(b) If we take $\gamma=\nu$ in (28) and make use of (19) and (24), we get

$$
\begin{gathered}
\int_{1}^{\infty}\left(x^{2}-1\right)^{-\frac{1}{2} \mu}\left(p^{2} x^{2}-1\right)^{-\frac{1}{2} \alpha} P_{\nu}^{-\alpha}(p x) P_{\nu}^{\mu}(x) d x \\
=2^{\mu+\alpha-2} p^{\mu-1} \frac{\Gamma\left(\frac{1}{2} \mu+\frac{1}{2} \alpha+\nu+\frac{1}{2}\right) \Gamma\left(\frac{1}{2} \mu+\frac{1}{2} \alpha-\nu-\frac{1}{2}\right) \Gamma\left(\frac{1}{2} \mu+\frac{1}{2} \alpha\right)}{\sqrt{\pi} \Gamma(\alpha-\nu) \Gamma(\alpha+\nu+1)}\left(p^{2}-1\right)^{\frac{1}{2}-\frac{1}{2} \mu-\frac{1}{2} \alpha} P_{-\nu-1}^{\frac{1}{2}-\frac{1}{2} \mu-\frac{1}{2} \alpha}\left(\frac{p^{2}+1}{2 p}\right),
\end{gathered}
$$

where $R(\mu)<1, R(\mu+\alpha+2 \nu+1)>0, R(\mu+\alpha-2 \nu-1)>0, R(\mu+\alpha)>0$ and $\left|1-p^{-2}\right|<1$.

(c) If we put $\gamma=\frac{1}{2}-\mu-\alpha$ in (28) and use the expansion [3, p. 125]

$$
\Gamma(1-\mu) P_{v}^{\mu}(z)=\left(\frac{z+1}{z-1}\right)_{2}^{\frac{1}{2 \mu}}{ }_{2} F_{1}\left(-v, 1+\nu ; 1-\mu ; \frac{1}{z}-\frac{1}{2} z\right),
$$


we get

$$
\begin{aligned}
& \int_{1}^{\infty}\left(x^{2}-1\right)^{-\frac{1}{2} \mu}\left(p^{2} x^{2}-1\right)^{-\frac{1}{2} \alpha} P_{t-\mu-\alpha}^{-\alpha}(p x) P_{\nu}^{\mu}(x) d x \\
& =2^{\mu+\alpha-2} p^{\mu-\frac{3}{2}} \frac{\Gamma\left(\frac{1}{2} \nu+\frac{3}{4}\right) \Gamma\left(-\frac{1}{2} \nu+\frac{1}{4}\right) \Gamma\left(\mu+\alpha+\frac{1}{2} \nu-\frac{1}{4}\right) \Gamma\left(\mu+\alpha-\frac{1}{2} \nu-\frac{3}{4}\right)}{\pi\left(\mu+2 \alpha-\frac{1}{2}\right) \Gamma\left(\frac{3}{2}-\mu\right)} \\
& \times\left(1-p^{2}\right)^{1-\frac{1}{2} \mu-\frac{1}{2} \alpha} P_{\frac{1}{2} \nu-\frac{1}{1}}^{1-\mu-\alpha}\left(2 p^{-2}-1\right),
\end{aligned}
$$

where $R(\mu)<1, R(1-2 \mu-2 \alpha)<R\left(-\nu-\frac{1}{2}\right)<1, R(\nu)<\frac{1}{2}$ and $\left|1-p^{-2}\right|<1$.

(d) If we take $\nu=\frac{1}{2}-\mu-\alpha$ in (28) and apply (15) we get

$$
\begin{aligned}
& \int_{1}^{\infty}\left(x^{2}-1\right)^{-\frac{1}{2} \mu}\left(p^{2} x^{2}-1\right)^{-\frac{1}{2} \alpha} P_{\gamma}^{-\alpha}(p x) P_{\frac{1}{2}-\mu-\alpha}^{\mu}(x) d x \\
& =2^{\mu+\alpha-2} \frac{\Gamma\left(\frac{1}{2} \gamma+\frac{3}{4}\right) \Gamma\left(\mu+\alpha+\frac{1}{2} \gamma-\frac{1}{4}\right) \Gamma\left(\frac{1}{4}-\frac{1}{2} \gamma\right) \Gamma\left(\mu+\alpha-\frac{1}{2} \gamma-\frac{3}{4}\right)}{\pi \Gamma(\alpha-\gamma) \Gamma(\alpha+\gamma+1)} \\
& \times p^{\mu-\frac{1}{2}}\left(p^{2}-1\right)^{\frac{1}{2}-\frac{1}{2} \mu-\frac{1}{2} \alpha} P_{-\frac{1}{2} \gamma-\frac{\alpha}{1}}^{1-\mu}\left(2 p^{2}-1\right),
\end{aligned}
$$

where $R(\mu)<1$, 步 $>R(\gamma)>-\frac{3}{2}, R\left(\mu+\alpha+\frac{1}{2} \gamma\right)>\frac{1}{4}, \quad R\left(\mu+\alpha-\frac{1}{2} \gamma-\frac{3}{4}\right)>0$ and $\left|1-p^{-2}\right|<1$.

(e) If we put $\nu=0$ in (28) and apply (22), (23) and (24) we get

$$
\begin{aligned}
\int_{1}^{\infty}(x-1)^{-\mu}\left(p^{2} x^{2}-1\right)^{-\frac{1}{2} \alpha} P_{\gamma}^{-\alpha}(p x) d x \\
\quad=\frac{\Gamma(\mu+\alpha+\gamma) \Gamma(\mu+\alpha-\gamma-1) \Gamma(1-\mu)}{\Gamma(\alpha-\gamma) \Gamma(\alpha+\gamma+1)} p^{\mu-1}\left(p^{2}-1\right)^{\frac{1}{1-\frac{1}{2} \mu-\frac{1}{2} \alpha} P_{-\gamma-1}^{1-\mu-\alpha}(p)} \\
\quad=\frac{\Gamma(\mu+\alpha+\gamma) \Gamma(\mu+\alpha-\gamma-1) \Gamma(1-\mu)}{\Gamma(\alpha-\gamma) \Gamma(\alpha+\gamma+1)} p^{\mu-1}\left(p^{2}-1\right)^{\frac{1}{2}-\frac{1}{2} \mu-\frac{1}{2} \alpha} P_{\gamma}^{1-\mu-\alpha}(p),
\end{aligned}
$$

where $R(\mu)<1, R(\mu+\alpha+\gamma)>0, R(\mu+\alpha-\gamma-1)>0$ and $\left|1-p^{-2}\right|<1$, by virtue of [3, p. 140]

$$
P_{-\gamma-1}^{\mu}=P_{\gamma}^{\mu}(z)
$$

(iii) If we start with $[9$, p. 179]

$$
\begin{aligned}
f(x) & =x^{\gamma-1} E\left[l ; \alpha_{r}: m+2 n ; \beta_{s}:(1 / x)^{2 n}\right] \\
& \fallingdotseq(2 / \pi)^{\frac{1}{2}}(2 \pi)^{1-n} 2^{\gamma-\frac{3}{2}} p^{1-\gamma} n^{\gamma-\frac{1}{2}} E\left[l ; \alpha_{r}: m ; \beta_{s}:\{p /(2 n)\}^{2 n}\right] \\
& =\psi(p),
\end{aligned}
$$

where $R(\gamma)>0, \beta_{m+\kappa+1}=(\gamma+1+2 \kappa) /(2 n), \beta_{m+n+1+\kappa}=(\gamma+2 \kappa) /(2 n) \quad(\kappa=0,1,2, \ldots, n-1)$, and

$$
\begin{aligned}
& x^{\mu-1} f(x)=x^{\mu+\gamma-2} E\left[l ; \alpha_{r}: m+2 n ; \beta_{s}:(1 / x)^{2 n}\right] \\
& \frac{K}{\bar{K}}(2 / \pi)^{1 / 2}(2 \pi)^{1-n} 2^{\mu+\gamma-\frac{s}{2}} n^{\mu+\gamma-\frac{3}{2}} p^{2-\mu-\gamma} \\
& \quad \quad \times E\left[l+2 n ; \alpha_{r}: m+2 n ; \beta_{s}:\{p /(2 n)\}^{2 n}\right] \\
&=\phi(p),
\end{aligned}
$$

where $\alpha_{l+\kappa+1}=\left(\mu+\gamma+\nu-\frac{1}{2}+2 \kappa\right) /(2 n), \alpha_{l+\kappa+n+1}=\left(\mu+\gamma-\nu-\frac{1}{2}+2 \kappa\right) /(2 n)(\kappa=0,1,2, \ldots, n-1)$, on applying the theorem and writing $\nu+\frac{1}{2}$ for $\nu$, we get

$$
\begin{array}{r}
\int_{1}^{\infty}\left(x^{2}-1\right)^{-\frac{1}{2} \mu} x^{-\gamma} E\left[l ; \alpha_{r}: m ; \beta_{s}:\left(\frac{p x}{2 n}\right)^{2 n}\right] P_{\nu}^{\mu}(x) d x \\
=(2 n)^{\mu-1} E\left[l+2 n ; \alpha_{r}: m+2 n ; \beta_{s}:\{p /(2 n)\}^{2 n}\right]
\end{array}
$$


where

$$
\alpha_{l+n+\kappa+1}=\frac{\mu+\gamma-\nu-1+2 \kappa}{2 n}, \alpha_{l+\kappa+1}=\frac{\mu+\gamma+\nu+2 \kappa}{2 n}, \beta_{m+\kappa+1}=\frac{\gamma+1+2 \kappa}{2 n}, \beta_{m+n+\kappa+1}=\frac{\gamma+2 \kappa}{2 n},
$$

$(\kappa=0,1,2, \ldots, n-1)$, and $R(\mu)<1, R(\mu+\gamma-\nu-1)>0, R(\mu+\gamma+\nu)>0, p \neq 0$, and $\quad|\arg p|<\frac{1}{2} \pi(l-m+1)$.

Two interesting particular cases of this result are given below.

(a) If we take $l=m+2, n=1, \alpha_{1}=\frac{1}{2}-\xi-\eta, \alpha_{2}=\frac{1}{2}-\xi+\eta, \alpha_{r+2}=\beta_{r} \quad(r=1,2, \ldots, m)$ in (39), substitute $x=\cosh \theta$ and use the relation

$$
E\left[\frac{1}{2}-\xi-\eta, \frac{1}{2}-\xi+\eta:: x\right]=\Gamma\left(\frac{1}{2}-\xi-\eta\right) \Gamma\left(\frac{1}{2}-\xi+\eta\right) x^{-\xi} e^{x / 2} W_{\xi, \eta}(x),
$$

we have

$$
\begin{aligned}
& \Gamma\left(\frac{1}{2}-\xi-\eta\right) \Gamma\left(\frac{1}{2}-\xi+\eta\right) \int_{0}^{\infty}(\sinh \theta)^{1-\mu}(\cosh \theta)^{-\gamma-2 \xi} e^{\frac{1}{p^{2}} \cosh ^{2} \theta} W_{\xi, \eta}\left(\frac{1}{4} p^{2} \cosh ^{2} \theta\right) P_{\nu}^{\mu}(\cosh \theta) d \theta \\
& \quad=p^{2 \xi} 2^{\mu-2 \xi-1} E\left[\frac{1}{2}-\xi-\eta ; \frac{1}{2}-\xi+\eta ; \frac{1}{2} \mu+\frac{1}{2} \gamma+\frac{1}{2} \nu ; \frac{1}{2} \mu+\frac{1}{2} \gamma-\frac{1}{2} \nu-\frac{1}{2}: \frac{1}{2} \gamma, \frac{1}{2} \gamma+\frac{1}{2}: \frac{1}{4} p^{2}\right], \ldots(41)
\end{aligned}
$$

where $R(\mu)<1, R(\mu+\gamma+\nu)>0, R(\mu+\gamma-\nu-1)>0, p \neq 0,|\arg p|<\frac{3}{2} \pi$.

By writing $z$ for $\frac{1}{4} p^{2}$, taking $\xi=\kappa-\lambda, \eta=-\frac{1}{4}, \mu=1-2 \lambda, \nu=2 m-\frac{1}{2}, \gamma=2 \lambda-2 \kappa+\frac{1}{2}$ and using (40), (24) and

we get $[8$, p. 601)]

$$
W_{1 l+i,-\frac{1}{2}}\left(\frac{1}{2} z^{2}\right)=2^{-1+l-\frac{1}{z}} z^{\frac{1}{3}} D_{l}(z),
$$

$$
\begin{array}{r}
\int_{0}^{\infty}(\sinh \theta)^{2 \lambda} e^{\frac{1}{2} \sinh ^{2} \theta} D_{2 \kappa-2 \lambda-\frac{1}{d}}\left(2 \frac{z}{z} \cosh \theta\right) P_{2 m-\frac{1}{2}}^{1-2 \lambda}(\cosh \theta) d \theta \\
=2^{-\lambda-\kappa-\frac{z}{2}} z^{-\lambda-\frac{1}{2}} \frac{\Gamma\left(\frac{1}{2}-\kappa+m\right) \Gamma\left(\frac{1}{2}-\kappa-m\right)}{\sqrt{\pi} \Gamma\left(\frac{1}{2}-2 \kappa+2 \lambda\right)} W_{k, m}(z),
\end{array}
$$

where $R(\lambda)>0, R\left(\frac{1}{2}-\kappa+m\right)>0, R\left(\frac{1}{2}-\kappa-m\right)>0, z \neq 0$ and $|\arg z|<\frac{3}{2} \pi$.

(b) If we take $l=2, m=1, \alpha_{1}=a, \alpha_{2}=b, \beta_{1}=c, n=1$ in (39) and apply

$$
E(a, b: c: x)=\frac{\Gamma(a) \Gamma(b)}{\Gamma(c)}{ }_{2} F_{1}\left(a, b ; c ;-\frac{1}{x}\right) \quad(x>1),
$$

and then write $-p^{2}$ for $\frac{1}{4} p^{2}$, we get

$$
\begin{aligned}
& \int_{1}^{\infty}\left(x^{2}-1\right)^{-\frac{1}{2} \mu} x_{2}^{-\gamma} F_{1}\left(a, b ; c ; p^{-2} x^{-2}\right) P_{\nu}^{\mu}(x) d x \\
& \quad=2^{\mu-1} \frac{\Gamma(c)}{\Gamma(a) \Gamma(b)} E\left[a, b, \frac{1}{2} \mu+\frac{1}{2} \gamma+\frac{1}{2} \nu, \frac{1}{2} \mu+\frac{1}{2} \gamma-\frac{1}{2} \nu-\frac{1}{2}: c, \frac{1}{2} \gamma+\frac{1}{2}, \frac{1}{2} \gamma:-p^{2}\right],
\end{aligned}
$$

where $R(\mu)<1, R(\mu+\gamma+\nu)>0, R(\mu+\gamma-\nu-1)>0$ and $|p|>1$.

Now putting $b=\frac{1}{2}+\frac{1}{2} \lambda+\frac{1}{2} m, a=1+\frac{1}{2} \lambda+\frac{1}{2} m, c=m+\frac{3}{2}$ in (45), using (24) and [3, p. 135]

we get

$$
\begin{aligned}
& 2^{-1-m} \sqrt{\pi} \Gamma(1+m+\lambda){ }_{2} F_{1}\left[1+\frac{1}{2} \lambda+\frac{1}{2} m, \frac{1}{2}+\frac{1}{2} \lambda+\frac{1}{2} m ; m+\frac{3}{2} ; z^{-2}\right] \\
& =\Gamma\left(m+\frac{3}{2}\right) z^{1+m+\lambda}\left(z^{2}-1\right)^{-1 \lambda} Q_{m}^{\lambda}(z),
\end{aligned}
$$

$$
\begin{aligned}
& \int_{1}^{\infty}\left(x^{2}-1\right)^{-1} \mu x^{m+\lambda-\gamma+1}\left(p^{2} x^{2}-1\right)^{-i \lambda} Q_{m}^{\lambda}(p x) P_{\nu}^{\mu}(x) d x \\
& =2^{\mu+\lambda-2} p^{-\lambda-m-1} E\left[\begin{array}{cc}
1+\frac{1}{2} \lambda+\frac{1}{2} m, \frac{1}{2}+\frac{1}{2} m+\frac{1}{2} \lambda, \frac{1}{2} \mu+\frac{1}{2} \gamma+\frac{1}{2} \nu, \frac{1}{2} \mu+\frac{1}{2} \gamma-\frac{1}{2} \nu-\frac{1}{2}: \\
m+\frac{3}{2}, \frac{1}{2} \gamma, \frac{1}{2} \gamma+\frac{1}{2}: & -p^{2}
\end{array}\right], \ldots
\end{aligned}
$$

where $R(\mu)<1, R(\mu+\gamma+\nu)>0, R(\mu+\gamma-\nu-1)>0$ and $|p|>1$. 
By putting $n=\lambda+l, \mu=-\nu=1-n, \gamma=2 m+2 n+2$ in (47) and applying the relations (13), (44) and (46) we get [6, p. 387]

$$
\int_{1}^{\infty} x^{l-m-n-1}\left(x^{2}-1\right)^{n-1}\left(p^{2} x^{2}-1\right)^{-\frac{1}{2} l-\frac{1}{2} n} Q_{m}^{l+n}(p x) d x=2^{n-1} n !\left(p^{2}-1\right)^{-\frac{1}{2} l} Q_{m+n}^{l}(p),
$$

where $R(n)>0, R(2 m+3)>0$ and $|p|>1$.

Also, if we take $\gamma=m+\lambda+1$ in (47) and use (44) we get (9).

I am very much indebted to Dr C. B. Rathie for his keen interest in the preparation of this paper.

\section{REFERENCES}

1. A. Erdélyi, Tables of integral transforms, Vol. I (New York, 1954).

2. A. Erdélyi, Tables of integral transforms, Vol. II (New York, 1954).

3. A. Erdélyi, Higher transcendental functions, Vol. I (New York, 1953).

4. S. Goldstein, Operational representation of Whittaker's confluent hypergeometric function and Weber's parabolic cylinder functions, Proc. London Math. Soc., (2) 34 (1932), 103-125.

5. T. M. MacRobert, Some integrals involving Legendre and Bessel functions, Quart. J. Math. (Oxford), (2) 42 (1940), 95-100.

6. T. M. MacRobert, Functions of a complex variable (London, 1954).

7. C. S. Meijer, Uber eine Erweiterung der Laplace-Transformation, Proc. Kun. Nederl. Akad. Wetensch., (5) 43 (1940), 599-608.

8. C. S. Meijer, Integraldarstellungen für Whittakersche Funktionen und ihre Produkte, Proc. Nederl. Akad. Wetensch., (5) 44 (1941), 599-605.

9. C. B. Rathie, A theorem in operational calculus and some integrals involving Legendre, Bessel and $E$-functions, Proc. Glasgow Math. Assoc., 2 (1956), 173-182.

Maharana Bhupal College

UDAIPUR 